\section{Die volumetrische Phosphorsäurebestimmung nach Pincus zur indirekten Bestimmung der Magnesia.}

Von J. W. Sprivaer,

Assistent im Chem. Laboratorium Dr. I. Pollak in Aussig a. E. (Eingeg. 14./4. 1919.)

Die Methode nach P i n c u s, welche zur Bestimmung der Phosphorsäure im Alkaliphosphat, Calciumphosphat usw. Anwendung findet und darauf boruht, daß sich die Phosphorsäure in neutralen oder schwach essigsauren, gewöhnlich ammonsalzhaltigen Phosphatlösungen mit dem Uran einer Uranylacetatlösung zu unlöslichem Uranylphosphat umsetzt

$$
\begin{gathered}
\mathrm{KH}_{2} \mathrm{PO}_{4}+\mathrm{UO}_{2}\left(\mathrm{C}_{2} \mathrm{H}_{3} \mathrm{O}_{2}\right)_{2}=\mathrm{KC}_{2} \mathrm{H}_{3} \mathrm{O}_{2}+\mathrm{H}_{4} \mathrm{C}_{2} \mathrm{O}_{2}+\mathrm{UO}_{2} \mathrm{HPO}_{4} \\
\mathrm{KH}_{2} \mathrm{PO}_{4}+\mathrm{UO}_{2}\left(\mathrm{C}_{2} \mathrm{H}_{3} \mathrm{O}_{2}\right)_{2}+\mathrm{NH}_{4} \mathrm{C}_{2} \mathrm{H}_{3} \mathrm{O}_{2}=\mathrm{KC}_{2} \mathrm{H}_{3} \mathrm{O}_{2} \\
+2 \mathrm{H}_{4} \mathrm{C}_{2} \mathrm{O}_{2}+\mathrm{UO}_{2} \mathrm{NH}_{4} \mathrm{PO}_{4}
\end{gathered}
$$

und der Uranylüberschuß durch Tüpfeln auf pulverisiertes Ferrocyankalium erkannt wird, kann wohl als bekannt vorausgesetzt werden (siehe Tr e a d w e II, II. Band S. 543-545), nichtsdestoweniger seien aber in der Folge die wichtigsten Zahlen für diese Methode angeführt, soweit sie für dip indirekte Magnesiabestimmung in Frage kommen. Erforderliche Lösungen:

Uranylacetatlösung. $35 \mathrm{~g} \mathrm{UO}\left(\mathrm{C}_{2} \mathrm{H}_{3} \mathrm{O}_{2}\right)$ zu $1 \mathrm{l}$.

Ammoniumacetatlösung. $100 \mathrm{~g} \mathrm{NH}_{4} \mathrm{C}_{2} \mathrm{H}_{3} \mathrm{O}_{2}$ und $100 \mathrm{ccm} \mathrm{C}_{2} \mathrm{H}_{4} \mathrm{O}_{2}$ $(1,04) \mathrm{zu} 1 \mathrm{l}$.

Kaliumphosphatlösung. $19,18 \mathrm{~g} \mathrm{KH}_{2} \mathrm{PO}_{4}\left(10 \mathrm{~g} \mathrm{P}_{2} \mathrm{O}_{5}\right)$ zu $1 \mathrm{l}$ oder

Calciumphosphatlösung. $5,463 \mathrm{~g} \mathrm{Ca}_{3} \mathrm{P}_{2} \mathrm{O}_{8}\left(2,5 \mathrm{~g} \mathrm{P}_{2} \mathrm{O}_{5}\right)$ mit wenig Salpetersäure zu 11.

Zur Titerstellung der Uranylacetatlösung verwendet man $500 \mathrm{ccm}$ Phosphatlösung, deren Richtigkeit oder $\mathrm{P}_{2} \mathrm{O}_{5}$-Gehalt gleichzeitig durch die Phosphorsäurebestimmung nach $W$ o y oder $B$. S c h m i t z festgestellt wurde in der Weise, da B man $10 \mathrm{ccm}$ Ammonacetatlösung zusetzt und so lange Uranylacetatlösung zufließen läßt, bis ein Tropfen mit festem Ferrocyankalium Braunfärbung zeigt. Hierauf wird zum Sieden erhitzt und weiter Uranylacetatlösung zugesetzt, bis nunmehr die nach dem Erhitzen verschwundene Braunfärbung wieder auftritt.

Je nachdem nun ein Alkaliphosphat oder Calciumphosphat vorliegt, wird die Titerstellung der Uranylacetatlösung mit Kalium. oder Calciumphosphat vorgenommen.

Bei Gegenwart von Eisen oder Aluminium, welche beide wohl meistens anwesend sind, liefert diese Methode keine guten Resultate, da die Phosphate derselben in Essigsäure unlöslich sind. Man muB demnach in einem solchen Falle die unlöslichen Phosphate von der Lösung trennen und gesondert nach einer der bekannten gewichtsanalytischen Phosphorsäurebestimmungsmethoden untersuchen, was logischerweise die doppelte Arbeit erfordert, da man bei sofortiger gravimetrischer Bestimmung schneller zum Ziele kommt.

Die indirekte Magnesiabestimmung beruht nun darauf, daß das nach der Fällung mit Phosphat nach B. S c h m i t $z$ oder W. G i b b s erhaltene Magnesiumammoniumphosphat in vorstehender Ammonacetatlösung gelöst und mit Uranylacetatlösung titriert wird.

$\mathrm{MgNH}_{4} \mathrm{PO}_{4}+\mathrm{UO}_{2}\left(\mathrm{C}_{2} \mathrm{H}_{3} \mathrm{O}_{2}\right)_{2}=\mathrm{UO}_{2} \mathrm{NH}_{4} \mathrm{PO}_{4}+\mathrm{Mg}\left(\mathrm{C}_{2} \mathrm{H}_{3} \mathrm{O}_{2}\right)_{2}$.

Hierbei ist selbstverständlich Hauptbedingung, daß die Magnesiafällung stets gleichmäßig vorgenommen wird, da erklärlicherweise eine Verunreinigung des Ammoniummagnesiumphosphats durch dreibasisches Magnesiumphosphat oder Monomagnesiumphosphat einen verschiedenen Phosphorsäuregehalt ergeben, und demnach auch bei der Umrechnung ein zu niedriger Magnesiawert gefunden würde.
Die besten Resultate wurden nach der Methode B. S c h m it z erhalten, und die Zusammensetzung des nach dieser Methode erhaltenen Magnesiumammoniumphosphats wurde bei mehreren Versuchen bis auf einige minimale Differenzen, welche als Analysenfehler anzusehen sind, stets gleichmäßig gefunden.

Die Fällungsmethode nach $\mathrm{S} \mathrm{ch} \mathrm{mitz}$ ist wohl bekannt, aber sie soll hier näher beschrieben werden, da damit gleichzeitig der Gesamtgang zur indirekten Bestimmung der Magnesia gegeben wird.

Die saure, ammonsalzhaltige Magnesiumsalzlösung wird mit einem Überschuß von Natrium- oder Ammoniumphosphat zum Sieden erhitzt, und die heiße Lösung sofort mit $1 / 3$ ihres Volumens an $10 \%$ igem Ammoniak versetzt. Nach dem Abkühlen wird filtriert, und das Magnesiumammoniumphosphat mit 21/2\% igciu Ammoniak gewaschen.

Dieses so erhaltene Magnesiumammoniumphosphat wird samt Filter in das ausgewaschene Glas zurückgebracht, mit 10-20 ccm Ammonacetatlösung und $50 \mathrm{ccm}$ Wasser versetzt und zum Sieden erhitzt. Hierauf wird mit Uranylacetatlösung in angegebener Stärke titriert, bis 1 Tropfen mit pulverisiertem Ferrocyankalium reagiert. Das sofortige Titrieren der heißen Lösung ist hier darum möglich, weil kein Calciumphosphat zugegen ist, und so eine Abscheidung von sekundärem Calciumphosphat nicht eintreten kann.

Die Anwendung dieser Methode scheint auch zur Bestimmung der Phosphorsäure in Phosphaten mit Eisen und Aluminium möglich, wenn man, statt das gefällte Magnesiumammoniumphosphat durch Glühen in Pyrophosphat überzuführen, mit Uranylacetatlösung titriert.

Die Titerstellung der Uranylacetatlösung zur Bestimmung der Magnesia erfolgt in der Weise, daß man eine Lösung mit bekanntem Magnesiagehalt in gleicher Weise behandelt wie die Probe, $d$. b. mit Phosphat nach B. S c h m i z fällt und das erhaltene Magnesiumammoniumphosphat titriert.

Wie bei allen Tüpfelmethoden ist es auch hier zur Erzielung guter Werte notwendig, beim Titrieren der Probe und des Titers gleiche Bedingungen, besonders gleiches Volumen einzuhalten.

Die Methode wurde natürlich vor ihrer praktischen Anwendung eingehend geprüft. Die Resultate der Bestimmung des Magnesiagehaltes in einigen Magnesiasalzen werden in folgendem angegeben.

Der Titer für die Uranylacetatlösung wurde mit Magnesiumammoniumphosphat, aus Magnesiumsulfat erhalten, ermittelt.

$10 \mathrm{~g} \mathrm{MgSO}_{4}, 7 \mathrm{aq}$. wurden zu $500 \mathrm{ccm}$ gelöst und $50 \mathrm{ccm}$ dieser Lösung nach der Methode B. S c h m it z mit Ammoniumphosphat gefällt.

Erhaltenes $\mathrm{Mg}_{2} \mathrm{P}_{2} \mathrm{O}_{7}=0,4504 \mathrm{~g}=0,1632 \mathrm{~g} \mathrm{MgO}$.

Die ebenfalls aus 50 ccm Lösung erhaltene Menge $\mathrm{MgNH}_{4} \mathrm{PO}_{4}$ wurde mit Uranylacetatlösung titriert, und es wurden $49,8 \mathrm{ccm}$ $\mathrm{UO}_{2}\left(\mathrm{C}_{2} \mathrm{H}_{3} \mathrm{O}_{2}\right)_{2}$ verbraucht:

Der Titer der Uranylacetatlösung berechnet sich demnach auf $\mathrm{MgO}$ mit $0,003277$.

theoretische Menge nach B. Schmitz Volumtr.

Magnesiumcarbonat . . 47,82\% $\mathrm{MgO} \quad 47,51 \% \mathrm{MgO} \quad 47,49 \%$

Magnesiumchlorid . . 25,56\% $\mathrm{Mg} \quad 25,49 \% \mathrm{Mg} \quad 25,41 \%$

Magnesiumhydroxyd . 6 69,11\% $\mathrm{MgO} \quad 69,00 \% \mathrm{MgO} \quad 68,98 \%$

Magnesiumoxyd . . . 60,34\% $\mathrm{Mg} \quad 60,21 \% \mathrm{Mg} \quad 60,25 \%$

Die Vorteile dieser Methode bestehen darin, daß man durch die schnell auszuführende Titration dic Zeit des Veraschens erspart und auch den häufig auftretenden Fehler unvollständiger Umwandlung des Magnesiumammoniumphosphats vermeidet.

Die Verabeitung der Uranrückstände macht die an und für sich jetzt etwas kostspielige Methode zum großen Teil billiger. [A. 67.] 